\title{
Chemical composition and DSC thermal properties of two species of Hylocereus cacti seed oil: hylocereus undatus and Hylocereus polyrhizus.
}

\begin{abstract}
Two types of pitaya (Hylocereus cacti) seeds (Hylocereus undatus and Hylocereus polyrhizus) were investigated in this study. The fatty acid, phenolic, tocopherol, and sterol contents of the extracted seed oil were analysed. The results showed that the pitaya seeds contained a high amount of oil (18.33-28.37\%). The three major fatty acids in the H. undatus seed oil (WFSO) and $\mathrm{H}$. polyrhizus seed oil (RFSO) were linoleic, oleic, and palmitic acids. The total tocopherol contents in the WFSO and RFSO were 36.70 and $43.50 \mathrm{mg} / 100 \mathrm{~g}$, respectively. The phytosterol compounds identified in the WFSO and RFSO were cholesterol, campesterol, stigmasterol, and $\beta$-sitosterol. Seven phenolic acid compounds were identified in the WFSO and RFSO, namely, gallic, vanillic, syringic, protocatechuic, p-hydroxybenzoic, p-coumaric, and caffeic acids. WFSO and RFSO can be differentiated by their Toff and Ton values in the DSC thermal curves. This study reveals that pitaya seed oil has a high level of functional lipids and can be used as a new source of essential oil.
\end{abstract}

Keyword: Hylocereus undatus; Hylocereus polyrhizus; Pitaya seed oil; Thermal properties; Tocopherol; Phytosterol; Phenolic acid. 